\title{
Prevalence of trachoma among children in East Jerusalem in 1980
}

\author{
S. BISHARA AND L. YANKO
}

From the Department of Ophthalmology, and the Jerusalem Institute for the Prevention of Blindness, Hadassah University Hospital and Hebrew University-Hadassah Medical School, Jerusalem, Israel

SUMMARY In an ophthalmic survey conducted in East Jerusalem during 1980 comprising 8896 nursery and primary school children aged 3 to 12 years not a single case of active trachoma could be detected. A similar study carried out in 1968, encompassing the same geographical area, revealed a prevalence of $6.4 \%$ among the school children and of $12.5 \%$ among the nursery school infants, while a subsequent survey performed in 1971, including the same population age group, showed a sharp decrease in the incidence of trachoma to a level as low as $1 \%$. This gradual and continuous decline in the prevalence of the disease towards its end point of apparent eradication was preceded by a marked improvement in the socioeconomic status and personal and public hygiene of the general population.

Endemic trachoma constitutes a major public health threat and is the chief cause of visual impairment and preventable blindness in certain developing countries. In fact this disease still remains a universal problem.' 'Despite the immense efforts invested in its eradication about 400 to 500 million individuals are afflicted with it. ${ }^{2}$ One of the major areas of the world where trachoma is as yet undefeated is the Middle East. ${ }^{3-5}$

Epidemiological studies have recorded a prevalence of $36 \%$ among the young population in certain parts of Saudi Arabia ${ }^{6}$ and of $60.1 \%$ in Abu-Dhabi. ${ }^{7}$ In 1922 active trachoma was prevalent in $72 \%$ of the Arab school children throughout Palestine, while in 1939 this incidence had decreased to $49 \%{ }^{8}$ In 1968 , during an ophthalmic survey in East Jerusalem. trachoma was found in $12.5 \%$ of nursery school infants and in $6.4 \%$ of primary school children. ${ }^{9}$ At this time an antitrachoma campaign was started, including treatment with antibiotic eye ointments. A subsequent study performed in 1971 including the same primary and nursery schools showed an incidence of only about $1 \%$ in this population group. 9

In view of recent improvements in social and medical conditions an additional epidemiological study, applying the same basic clinical designs and encompassing the nursery and primary schools of the

Correspondence to S. Bishara. MD. Department of Ophthalmology. Hadassah University Hospital. PO Box 12000. Jerusalem, 91 120 Israel. previous 2 surveys conducted by Maythar in 1968 and 1971, was undertaken to assess the current frequency and trend of trachoma.

\section{Subjects and methods}

Of the 26 governmental primary and nursery schools in East Jerusalem and environs 24 randomly chosen schools, with an attendance of 9364 pupils aged between 3 and 12 years, form the basis of this report. The eyes were examined with the aid of a 2 times magnifying loupe under proper illumination. The eyelids, the cornea, the corneoscleral limbus, and the bulbar, tarsal, and forniceal conjunctiva were thoroughly checked. Exploration of the last was achieved by flipping up the upper eyelid with 2 fingers while the child was looking down and at the same time exerting pressure on the globe from below, thus causing protrusion of the forniceal conjunctiva, where the follicles of trachoma usually appear first.

The criteria for the diagnosis and classification of the disease were based on the scoring system established by the WHO Expert Committee ${ }^{10}$ and the WHO Fourth Scientific Group ${ }^{11}$ and modified by us (Table 1). Patients with suspected trachoma, screened out during the examination, underwent slit-lamp biomicroscopy assessment and conjunctival scraping. The smears were Giemsa stained and tested for the presence of inclusion bodies. This latter method has remained the easiest technique available, and is a 
Table 1 Criteria for classification and diagnosis of trachoma

\begin{tabular}{|c|c|c|c|c|c|}
\hline \multirow{2}{*}{$\begin{array}{l}\text { Clinical oculiar } \\
\text { findings }\end{array}$} & \multicolumn{5}{|l|}{ Scoring } \\
\hline & 0 & $l$ & $\underline{2}$ & 3 & 4 \\
\hline Conjunctival hvperaemia & Absent & Mild & Moderate & Severe & Hacmorrhagic \\
\hline Conjunctival follicles & Absent & $<5$ & $5-10$ & $11-15$ & $>15$ \\
\hline Conjunctival papillae & Absent & Minimal & Moderate & Pronounced & - \\
\hline Corneal infiltrate & Absent & $\begin{array}{l}<1.5 \text { Thickened } \\
\text { cornea }\end{array}$ & $\begin{array}{l}>1.5 \text { Thickened } \\
\text { cornea }\end{array}$ & - & - \\
\hline Pannus & Absent & $\begin{array}{l}<1 \mathrm{~mm} \text { in the } \\
\text { cornea }\end{array}$ & $\begin{array}{l}1-1.5 \mathrm{~mm} \text { in the } \\
\text { cornea }\end{array}$ & $\begin{array}{l}>1.5 \mathrm{~mm} \text { in the } \\
\text { cornea }\end{array}$ & - \\
\hline Herbert's pits & Absent & $1-3$ & $>3$ & $\begin{array}{l}\text { Entire upper lunula } \\
\text { involved }\end{array}$ & Cornea encircled \\
\hline Conjunctival scar & Absent & Mild & Moderate & Severe & $\begin{array}{l}\text { Trichiasis and/or } \\
\text { entropion }\end{array}$ \\
\hline
\end{tabular}

widely accepted mode for epidemiological screening for trachoma. ${ }^{12}$

\section{Results}

Of a total of 9364 pupils attending the schools included in the survey 8896 participated in the study and were screened for trachoma. On initial examination only 3 children displayed follicular infiltrate of the upper palpebral and forniceal conjunctiva in both eyes which, in spite of normal corneas and limbi, was suggestive of active trachoma. Biomicroscopy and laboratory tests failed to confirm the diagnosis. In one of the children a bacterial infection was diagnosed and successfully treated with antibiotics. Examination for the presence of inclusion bodies performed in 37 additional children with clinical signs of follicular or papillary hypertrophy due to nonspecific or allergic conjunctivitis proved negative.

\section{Discussion}

In the present study, undertaken 10 years after the previous studies by Maythar. ${ }^{9}$ and involving the same nursery and elementary schools as those of Maythar's survey, when 8896 of $9364(95 \%)$ regularly attending pupils were examined, no cases of active trachoma could be detected. The absence of the 468 children on the screening days was due to a variety of reasons and does not represent parental noncompliance with the survey itself. Moreover the number of absentees compared with that of the examined children is too small to affect the results statistically $(p=0 \cdot 0003)$.

The findings of the current survey unequivocally demonstrate that the incidence of trachoma has steadily declined, approaching zero, thus reaching the desired end point in the fight for eradication of the disease in the geographical area at issue. Considering the fact that no preventive medical treatment has been administered in the last decade-except a routine sanitary supervision-it is reasonable to assume that these satisfactory results are due to the constantly improving hygienic and socioeconomic conditions.

The third report of the WHO Expert Committee ${ }^{10}$ listed the following environmental factors as influencing the frequency of trachoma: race, climate. insect vectors, population density, diet and nutrition. cultural and social customs, general economic level. extrinsic contacts, educational level. and the presence of other ocular or general diseases.

Among all these factors only the first 2 and the social customs have remained unchanged in East Jerusalem, while all other conditions enumerated above have been vastly improved. The marked progress in the socioeconomic conditions and educational levels have been conducive to a decrease in population density and as well as to the enhancement of the dietary and nutritional state. At the same time the tremendous decrease of insect vectors, which are mainly responsible for the extrinsic contact, has contributed to the abolition of ocular discharge transmitted by flies. ${ }^{13}$ The present availability of running water in almost every home and school, as well as the diminished incidence of bacterial conjunctivitis. largely facilitated the defeat of trachoma.

We are greatly indebted to Professor I. C. Michaelson and Dr B. Mavthar for their valuable guidance.

\section{References}

1 World Health Organisation. Field Methods for the Control of Trachoma. Tarizzo ML. ed. 1973: 11. 46.

2 World Health Organisation. Prevention of blindness. WHO Chron 1973: 27: 21-7.

3 Maichuk IF. Methods for mass trachoma control in the Middle East. Rev Int Trach Pathol Ocul Trop Subtrop 1975; 52: 33-49.

4 Daghfous T. (1976). Trachoma-a public health problem: report. part I. Rev Int Trach Pathol Ocul Trop Subtrop 1976: 52: 59-81.

5 Dawson C. Trachoma-a public health problem: report. part II. Rev Int Trach Pathol Ocul Trop Subtrop 1976: 52: 82-91). 
6 Bobb AA Jr, Nichols RL. Influence of environment on clinical trachoma in Saudi Arabia. Am J Ophthalmol 1969; 67: 235-43.

7 Maichuk IF. Trachoma treatment in areas of high prevalence. Rev Int Trach Pathol Ocul Trop Subtrop 1973; 50: 55-66.

8 Feigenbaum A. Fifty years of ophthalmology in Israel. Harefuah 1945; 29: 161-71.

9 Maythar B. Jerusalem seminar on the prevention of blindness. 2nd Conference of the International Society of Geographical Ophthalmology: 1971: 27-8.
10 World Health Organisation. Expert Committee on Trachoma. 3rd Report. WHO Tech Rep Ser 1962: 234.

11 World Health Organisation. Fourth WHO Scientific Group. WHO Tech Rep Ser 1966: 330.

12 Yoneda C, Dawson CR, Daghfous T. et al. Cytology as a guide to the presence of chlamydial inclusions in Giemsa-stained conjunctival smears in severe endemic trachoma. $\mathrm{Br} J$ Ophthalmol 1975; 59: 116-24.

13 Jones BR. Changing concepts of trachoma and its control. Trans Ophthalmol Soc UK 1980; 100: 25-9. 